\title{
Opioid receptors revealed
}

Two more structures join the parade of once-intractable proteins.

\section{BY LIZZIE BUCHEN}

$\mathrm{O}$ $\mathrm{n}$ a small table in his office at the Scripps Research Institute in La Jolla, California, Ray Stevens spreads out a sheet of paper covered with colourful branched lines, each sprouting and thinning before terminating in an esoteric code.

"This is the dream," he declares.

The intricate diagram represents the largest family of receptor proteins encoded in the genome - the G-protein-coupled receptors (GPCRs), ubiquitous cellsurface molecules that are activated by light, odours, hormones and neurotransmitters. Stevens wants to determine the atomic structures of receptors on all branches of the tree. This week, that goal moved two receptors closer: Stevens's group has solved the atomic structure of the $\kappa$-opioid receptor $(\kappa-\mathrm{OR})^{1}$, and a team led by Brian Kobilka at Stanford University in California has solved the medically crucial $\mu$-opioid receptor $(\mu-\mathrm{OR})^{2}$. The structures, published in Nature, bring the tally of GPCR structures solved this year alone up to five.

Ten years ago, Stevens's dream would have seemed a hopeless fantasy - the complex, dynamic molecules had confounded relentless attempts to expose their atomic secrets by X-ray crystallography. But in 2007, several technological breakthroughs brought the drought to an end ${ }^{3}$, and the structures began to trickle out, with one or two solved nearly every year. Now, it seems the flood is approaching. Stevens's and Kobilka's groups each have another structure ready to go, on top of the five GPCRs they have already published this year ${ }^{4-6}$. Stevens predicts several more from his lab by the end of the year.

The opioid receptors are hot targets. Opioid drugs, including morphine, codeine and oxycodone, are invaluable in medicine for their powerful painkilling and sedative effects. But they are also highly addictive, an enormous liability that limits their medical use. Their ability to produce a strong sense of euphoria also leads to rampant recreational use of prescription drugs and their more potent and deadly derivative, heroin. The majority of their effects, including analgesia, euphoria and sedation, are mediated by the $\mu-O R$, although most opioid drugs are promiscuous, activating $\kappa$-OR and other opioid receptors as well. Understanding the molecular structure of these receptors is crucial for designing drugs that exert the beneficial actions of opioids but lack the unwanted side-effects.

But opioid receptors, like other GPCRs, had long dodged the scrutiny of X-ray crystallography. The technique requires relatively

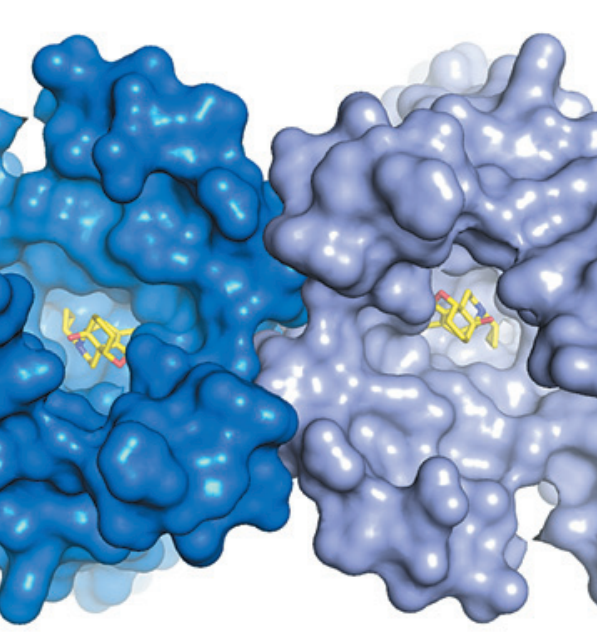

When crystallized with a ligand (yellow), $\mu$-opioid receptors form an intimate pair.

large amounts of purified protein; for proteins nestled in cell membranes, such as GPCRs, that means carefully extracting the molecules from their fatty environment while maintaining their delicate shapes. Identical copies of the protein must then be packed so tightly that they form a crystal. This step is particularly difficult for GPCRs, which have floppy loops that are resistant to staying in place and conforming to a regular crystal lattice.

But one by one, the bottlenecks were circumvented. Kobilka, who had worked on crystallizing the $\beta_{2}$-adrenergic receptor ( $\left.\beta_{2} \mathrm{AR}\right)$ for more than 20 years, discovered that he could quiet the most restive loop by replacing it with a protein called T4L. In 2007, he and Stevens both crystallized the $\beta_{2} \mathrm{AR}$ using T4L and a medium called the lipidic cubic phase ${ }^{7,8}$. Since then, all but one of the solved GPCRs have been cracked using this approach.

The $\mu$-OR and the $\kappa$-OR structures reveal that the binding pockets of both receptors are large and gaping, which Stevens suggests could explain how the $\mathrm{\kappa}-\mathrm{OR}$ recognizes such a diverse set of molecules. Kobilka and his colleague Sébastien Granier, now a molecular pharmacologist at the French National Institute of Health and Medical Research in Montpellier, say that the openness of the $\mu$-OR binding site may also explain why the actions of opioids can turn on so quickly and be rapidly reversed. Heroin overdoses, for example, can be quickly treated with opioid antagonists such as naloxone.

Kobilka and his colleagues also discovered that the $\mu$-OR crystallized in tightly linked pairs, complementing several other lines of evidence that the receptor pairs with itself and with other opioid receptors in the body. These pairings may mediate the various effects of opioid drugs.

Philip Portoghese at the University of Minnesota in Minneapolis, who has been studying opioid receptors since the $1960 \mathrm{~s}$, is thrilled with the structures. "When I first started in the opioid field, crystallographic determination of any receptor was viewed as science fiction," he says. "Never in my wildest dreams did I think I would live to see the coordinates of not one, but two opioid receptors." Portoghese points out, however, that both receptors were crystallized in their inactivated states, which he says may limit the value of the structures in the hunt for safer painkillers.

Nor are crystal structures the be-all and end-all for rational drug design. GPCRs are not static; they writhe and twist, and crystal structures don't capture this crucial dynamism, which may be critical for designing drugs that can join in this dance. Kobilka, Stevens and many others are complementing crystallography with techniques that can capture protein movement, such as nuclear magnetic resonance and molecular-dynamics modelling.

On Stevens's website, where the tree of dreams is prominently displayed, 14 victorious red flags mark solved GPCRs, and more will follow very soon. It took at least 20 years of dogged devotion to solve the $\beta_{2} \mathrm{AR}-$ but today, when someone visits Stevens's lab to learn about cystallography, he has them crystallize the once-uncrystallizable protein as an introductory exercise. It takes three days. .

1. Wu, H. et al. Nature http://dx.doi.org/10.1038/ nature10939 (2012).

2. Manglik, A. et al. Nature http://dx.doi.org/10.1038/ nature10954 (2012).

3. Buchen, L. Nature 476, 387-390 (2011)

4. Haga, K. et al. Nature 482, 547-551 (2012)

5. Kruse, A. C. et al. Nature 482, 552-556 (2012)

6. Hanson, M. A. et al. Science 335, 851-855 (2012).

7. Rosenbaum, D. M. et al. Science 318, 1266-1273 (2007).

8. Cherezov, V. et al. Science 318, 1258-1265 (2007). 